\title{
Thermal Perception Abnormalities Can Predict Diabetic Kidney Disease in Type 2 Diabetes Mellitus Patients
}

\author{
Wei-Ching Fang ${ }^{a}$ Kuei-Mei Chou ${ }^{b, c}$ Chiao-Yin Sun ${ }^{c, d}$ Chin-Chan Lee ${ }^{c, d}$ e \\ I-Wen Wu ${ }^{c, d}$, e Yung-Chang Chen ${ }^{c, d, e}$ Heng-Chih Pan ${ }^{d, e}$ \\ aDepartment of Family Medicine, Linkou Chang Gung Memorial Hospital, Taoyuan, Taiwan; \\ bDivision of Endocrinology and Metabolism, Department of Internal Medicine, Keelung Chang \\ Gung Memorial Hospital, Keelung, Taiwan; ' Chang Gung University College of Medicine, \\ Taoyuan, Taiwan; 'Division of Nephrology, Department of Internal Medicine, Keelung Chang \\ Gung Memorial Hospital, Keelung, Taiwan; 'Community Medicine Research Center, Keelung \\ Chang Gung Memorial Hospital, Keelung, Taiwan
}

\section{Keywords}

Thermal perception abnormalities - Quantitative sensory test - Diabetic kidney disease - Type 2 diabetes - Diabetic neuropathy

\begin{abstract}
Background: Previous studies have illustrated clinical associations between diabetic peripheral neuropathy (DPN) and diabetic kidney disease (DKD). Quantitative sensory testing (QST) can accurately detect thermal perception abnormalities and aid in the early diagnosis of asymptomatic small-fiber DPN in patients with type 2 diabetes. The aim of this study was to determine the predictive value of thermal perception abnormalities by QST to detect DKD. Methods: We prospectively enrolled 432 patients with type 2 diabetes (50.2\% male, mean age 57.2 years, and average duration of diabetes 9.9 years) at our hospital between 2016 and 2017. Demographic and clinical data of the patients were recorded and analyzed. Diagnosis and staging of DKD were determined by urinary albumin excretion rate and estimated glomerular filtration rate. The presence of thermal perception abnormalities was determined by QST. Multiple logistic regression and receiver operating characteristic (ROC) analyses were performed to investigate the relationships between thermal perception abnormalities and DKD in these patients. Results: In multiple regression analysis, abnormal cold perception in the lower limbs was associated with an increased risk of advanced DKD. Area under the ROC curve analysis revealed that four-limb cold perception abnormalities had the best discriminatory power
\end{abstract}

Wei-Ching Fang and Kuei-Mei Chou contributed equally to the manuscript. 
$(0.741 \pm 0.053)$ to predict advanced DKD. Conclusions: Our results demonstrate the value of using thermal perception abnormalities to identify patients with type 2 diabetes also at risk of DKD.

\section{Introduction}

Type 2 diabetes mellitus (T2DM) is one of the most important health problems worldwide and is associated with a wide variety of macrovascular and microvascular complications and increased morbidity and mortality [1-4]. Diabetic peripheral neuropathy (DPN) and diabetic nephropathy are 2 major chronic microvascular complications in T2DM patients and can lead to serious consequences including foot ulceration, lower extremity amputation, and renal failure. Thus, screening and early diagnosis of these T2DM complications are critical to provide an opportunity for effective interventions and treatment to prevent progression to more advanced stages $[5,6]$.

DPN is characterized by pain, tingling, and numbness, with prevalence rates ranging from 30 to $50 \%$ [7]. It is often associated with many comorbidities, including cognitive impairment, depression, autonomic neuropathy, peripheral artery occlusive disease (PAOD), nephropathy, retinopathy, cardiovascular disease, and medial arterial calcification $[8,9]$. The diagnosis of DPN is most often made based on suggestive clinical symptoms and neurologic tests. However, many patients with DPN have no obvious neuropathic symptoms despite showing evidence of neurologic deficits on nerve conduction studies (NCSs) or electromyography. NCSs are considered to be the gold standard to diagnose DPN, but they cannot be performed in all diabetic patients due to their difficulty to conduct, time-consuming, and cost $[10,11]$. In addition, NCSs only assess the function of large myelinated fast-conducting Aa and Ab fibers, and the test is usually normal if diabetic neuropathy predominately involves small-fiber neuropathy [12]. Due to the insidious nature of symptoms, DPN is often not diagnosed in the early stages. The severity of thermal perception abnormalities can reflect the extent of smallfiber neuropathy [13]. Quantitative sensory testing (QST) is a cheaper, portable, painless, and easy-to-use tool that can accurately detect thermal perception abnormalities. It is suggested that thermal perception abnormalities screening by QST can aid in the early diagnosis of asymptomatic small-fiber DPN [14].

Diabetic kidney disease (DKD) has been associated with a rapid progression in renal impairment and is now the most significant etiology of chronic kidney disease (CKD) and endstage renal disease globally. The progression of DKD can be attributed to not only diabetic nephropathy, but also some other diabetes-related comorbid conditions, such as neurologic bladder, frequent urinary tract infection, macrovascular angiopathy, and adverse effects of medication. Patients with advanced DKD are at high risk of kidney failure and death. A recent study had showed that estimated glomerular filtration rate (eGFR) $<45 \mathrm{~mL} / \mathrm{min} / 1.73 \mathrm{~m}^{2}$ (CKD stage $3 \mathrm{~b}$ ) at the time of nephrologist referral was an important risk factor for adverse outcomes in diabetes patients. Fortifying timely recognition and further optimization of nephroprotection measures for patients at risk of advanced DKD is an important issue in patients with diabetes [15-17].

Previous studies have indicated that DKD is significantly associated with DPN, and that patients with DPN have a higher incidence of albuminuria and lower eGFR than patients without DPN [18, 19]. Sheen et al. [9] had reported that thermal perception abnormalities of the lower limbs by QST are significantly associated with lower eGFR and higher albuminuria in type 2 diabetes. Given that the chronology of the occurrence of DPN and DKD in patients with T2DM is still unclear, we hypothesized that the severity of thermal perception abnor- 
mality of the 4 limbs by QST can help to detect patients at risk of kidney failure. Therefore, in this study, we aimed to determine the predictive value of thermal perception abnormality to detect the presence of advanced DKD in patients with T2DM.

\section{Materials and Methods}

\section{Study Design and Population}

This study was performed between October 2016 and December 2017 at Chang Gung Memorial Hospital, Keelung, Taiwan. Patients with T2DM between the ages of 18 and 70 years who attended an outpatient clinic in the hospital were recruited for the study. The exclusion criteria were patients with a urinary tract infection over the past 3 months, poorly controlled hypertension (HTN), thyroid function disorder, pregnancy, malignancy, liver cirrhosis, polycystic kidney disease, end-stage renal disease, systemic lupus erythematosus, and confirmed neuropathy owing to nondiabetic causes (alcoholism, nutrition deficiency, or uremia). All of the patients completed a survey including demographic information, common biochemical parameters, and assessments of macro- and microangiopathies. All of the study subjects were treated according to the American Diabetes Association diabetes mellitus treatment guideline [20].

\section{Definitions}

The presence of DKD was identified by assessing albuminuria and eGFR. Albuminuria was identified by calculating urine albumin/creatinine ratio (UACR, $\mathrm{mg} / \mathrm{g}$ ) in first-voided spot urine samples, and eGFR $\left(\mathrm{mL} / \mathrm{min} / 1.73 \mathrm{~m}^{2}\right)$ was calculated using the Modification of Diet in Renal Disease study equation [21,22]. The patients were defined as having advanced DKD if they had an eGFR $<45 \mathrm{~mL} / \mathrm{min} / 1.73 \mathrm{~m}^{2}[16,23]$. Coronary artery disease and cerebral vascular accidents were recorded by chart review. PAOD was diagnosed according to the ankle-brachial index (ABI) and was defined as the lowest values of right and left ABIs as measured by Doppler ultrasound. ABI is a measure of the blood pressure in the arteries supplying legs relative to central, aortic pressure (approximated by measuring the blood pressure in the arm). The right and left ABIs were determined as the right and left ankle systolic pressures divided by the highest brachial systolic pressure, respectively. PAOD was defined as an ABI $\leq 0.9$ or $>1.3$ [24]. Retinopathy was diagnosed based on the findings of an ophthalmic examination, including the best-corrected visual acuity and fundus examination by indirect ophthalmoscopy conducted by a retinal specialist. Macular edema was judged to be present if retinal thickening or hard exudates were detected at or within a 1-disc diameter of the center of the macula. Severity of retinopathy was classified according to the most severe changes in the worst-affected eye [25]. Neuropathy was judged to be present based on the following: (1) clinical symptoms of burning pain, paresthesia, hyperesthesia, tingling, and painful cramps involving the lower limbs; and (2) diminished fine touch sensation and impaired vibratory sensation based on the findings of nerve tests, including pinprick, vibration sensation, 10-g monofilament tests, and absence of Achilles reflex. In brief, pinprick sensation was measured using sterile Neurotip (Owen Mumford, Oxford, UK) 4 times on the same site. Vibration testing by the on-off method was conducted using a $128-\mathrm{Hz}$ tuning fork applied to the bony prominence just proximal to the nail bed on the dorsal side of the big toe. The patient reported perception of both the start of the vibration sensation and the cessation on dampening, conducted twice on each toe. Vibration testing by the timed method is performed by the patient reporting the time at which vibration is reduced to an undetectable level. The tuning fork was then applied to the back of the phalanx of the examiner's thumb. Then, the time (in seconds) at which the vibration sensation is weakened from the sides to beyond the 
examiner's perception was added to provide a score. The Vibration Perception Threshold test is quantitatively measured by a limit method using Medoc equipment (Medoc Advanced Medical Systems, Durham, NC, USA). Monofilament tests were performed bilaterally using a 10-g monofilament. First, a reference stimulation is applied to the forehead or sternum. With the patients' eyes closed, the monofilament is applied to the area just proximal to the nail bed on the noncallused dorsal side of the big toe using a smooth motion: tou ching the skin, bending the monofilament for a full second, and then lifting it from the skin. This maneuver was repeated 4 times per foot in a random arrhythmic manner. Each test was performed by an examiner blinded to results of all other examinations $[20,26]$. Doppler ultrasound, fundoscopic examinations, and nerve tests were routinely performed when the subjects were enrolled in this study [20].

\section{Determination of Thermal Perception Abnormalities by QST}

QST was performed using a Medoc device (TSA2001/VSA3001) following previously published procedures. All measurements were performed on the thenar eminence of the dominant hand and the lateral distal aspect of the foot dorsum of the same side. The adaptation temperature of the probe was $32^{\circ} \mathrm{C}$, and its contact area was $30 \times 46 \mathrm{~mm}^{2}$. Using the method of limits, a threshold was determined as the average of 4 successive stimuli for cold and warm sensations. Probe temperature change rates of $1^{\circ} \mathrm{C}$ were used for cold and warm thresholds. Five seconds elapsed before switching to the next sensory stimuli of the nonpainful modalities. All of the samples were measured twice, and the mean value was used for further statistical analysis $[9,27]$. Upper limb warmth perception abnormality (ULWPA) was defined as abnormal warmth perception in the right or left upper limbs ( $0-2$ points). Upper limb cold perception abnormality (ULCPA) was defined as abnormal cold perception in the right or left upper limbs (0-2 points). Lower limb warmth perception abnormality (LLWPA) was defined as abnormal warmth perception in the right or left lower limbs (0-2 points). Lower limb cold perception abnormality (LLCPA) was defined as abnormal cold perception in the right or left lower limbs. Upper limb warmth and cold perception abnormality (ULWCPA) was defined as a combination of both warmth and cold perception abnormalities in the upper limbs (0-4 points). Lower limb warmth and cold perception abnormality (LLWCPA) was defined as a combination of both warmth and cold perception abnormalities in the lower limbs (0-4 points). Four-limb warmth perception abnormality (FLWPA) was defined as abnormal warmth perception in the right or left/upper or lower limbs (0-4 points). Four-limb cold perception abnormality (FLCPA) was defined as abnormal cold perception in the right or left/upper or lower limbs (0-4 points). Four-limb warmth or cold perception abnormality (FLWCPA) was defined as a combination of both warmth and cold perception abnormalities in all 4 limbs (0-8 points).

\section{Statistical Analysis}

Continuous data were expressed as means and standard deviations, and categorical data were expressed as percentages. In the primary analysis, patients with and without advanced DKD were compared. Continuous variables were tested for normal distribution using the Kolmogorov-Smirnov test. Student's $t$ test was used to compare the mean values of continuous variables and normally distributed data; in other cases, the Mann-Whitney $U$ test was used. Categorical data were tested using the $\chi^{2}$ test. We assessed the risk factors for advanced DKD using univariate analysis, and the variables that were statistically significant $(p<0.05)$ in the univariate analysis were included in the multivariate analysis. A multiple logistic regression model and backward elimination of data were used to analyze these variables.

Correlations between paired groups of variables were assessed using Spearman's rank correlation analysis. Discrimination was calculated using the area under receiver operating characteristic (AUROC) curves. The AUROC values were compared using a nonparametric 
Table 1. Baseline characteristics of participants

\begin{tabular}{|c|c|c|c|c|}
\hline & Total $(n=432)$ & $\begin{array}{l}\text { eGFR } \geq 45 \\
(n=379)\end{array}$ & $\begin{array}{l}\text { eGFR <45 } \\
(n=53)\end{array}$ & $p$ value \\
\hline \multicolumn{5}{|l|}{ Demographics } \\
\hline Age, years & $57.2 \pm 10.3$ & $59.3 \pm 9.5$ & $66.2 \pm 6.7$ & $<0.001$ \\
\hline DM duration, months (median) & $118.3 \pm 89.9$ & $110.3 \pm 85.7$ & $175.1 \pm 97.4$ & $<0.001$ \\
\hline Male gender, $n(\%)$ & $217(50.2)$ & $193(50.9)$ & $24(45.3)$ & NS $(0.442)$ \\
\hline BMI & $27.4 \pm 4.6$ & $27.3 \pm 4.6$ & $27.9 \pm 4.5$ & NS (0.394) \\
\hline HTN, $n(\%)$ & $270(62.5)$ & $219(57.8)$ & $51(96.2)$ & $<0.001$ \\
\hline $\mathrm{CAD}, n(\%)$ & $42(9.7)$ & $34(9.0)$ & $8(15.1)$ & NS (0.127) \\
\hline CVA, $n(\%)$ & $22(5.1)$ & $17(4.5)$ & $5(9.4)$ & NS (0.161) \\
\hline PAOD, $n(\%)$ & $5(1.2)$ & $2(0.5)$ & $3(5.7)$ & 0.015 \\
\hline Retinopathy, $n(\%)$ & $76(17.6)$ & $57(15.0)$ & $19(35.8)$ & 0.001 \\
\hline Neuropathy, $n(\%)$ & $103(23.8)$ & $81(21.4)$ & $22(41.5)$ & 0.001 \\
\hline Smoking, $n(\%)$ & $104(24.1)$ & $93(24.5)$ & $11(20.8)$ & NS (0.494) \\
\hline Family history of DM, $n(\%)$ & $110(23.1)$ & $100(26.4)$ & 10 (18.9) & NS (0.180) \\
\hline \multicolumn{5}{|l|}{ Laboratory results } \\
\hline $\mathrm{eGFR}, \mathrm{mL} / \mathrm{min} / 1.73 \mathrm{~m}^{2}$ & $60.1 \pm 9.5$ & $84.5 \pm 22.6$ & $30.7 \pm 10.5$ & $<0.001$ \\
\hline UACR, mg/g (median) & $318.3(24.0)$ & $164.0(19.0)$ & $1,261.8(521.0)$ & 0.001 \\
\hline Total cholesterol, mg/dL (median) & $147.0(142.0)$ & $185.1(170.0)$ & $177.6(167.5)$ & NS (0.676) \\
\hline HDL cholesterol, mg/dL & $45.3 \pm 21.9$ & $45.7 \pm 22.8$ & $42.4 \pm 14.3$ & NS (0.305) \\
\hline LDL cholesterol, mg/dL & $99.9 \pm 36.1$ & $101.3 \pm 36.7$ & $89.7 \pm 29.9$ & 0.028 \\
\hline Triglycerides, mg/dL (median) & $180.9(147.0)$ & $171.8(142.0)$ & $247.5(183.0)$ & 0.042 \\
\hline ALT & $29.4 \pm 20.4$ & $30.3 \pm 21.2$ & $22.8 \pm 11.4$ & $<0.001$ \\
\hline $\mathrm{HbA1C}, \%$ & $7.8 \pm 1.7$ & $7.8 \pm 1.7$ & $8.2 \pm 2.1$ & NS (0.118) \\
\hline HS-CRP, mg/L (median) & $3.1[2.0]$ & $3.2[2.0]$ & $2.8[2.0]$ & NS (0.808) \\
\hline Homocysteine & $13.7 \pm 6.7$ & $11.2 \pm 3.8$ & $21.0 \pm 8.9$ & NS (0.191) \\
\hline
\end{tabular}

Values in bold are statistically significant $(p<0.05)$. ALT, alanine transaminase; BMI, body mass index; HTN, hypertension; CAD, coronary artery disease; CVA, cerebrovascular accident; DM, diabetes mellitus; eGFR, estimated glomerular filtration rate; HbA1C, glycated hemoglobin; HDL, high-density lipoprotein; HS-CRP, high-sensitivity C-reactive protein; LDL, low-density lipoprotein; PAOD, peripheral artery occlusive disease; UACR, urine albumin-to-creatinine ratio.

approach. AUROC analysis was also used to calculate the cutoff values, sensitivity, specificity, and overall correctness. Finally, cutoff points were calculated by calculating using the Youden index (sensitivity + specificity - 1). All statistical analyses were 2-tailed, and a $p$ value of $<0.05$ was considered to be statistically significant. All data were analyzed using the Statistical Package for Social Sciences software, version 20.0 for Windows (SPSS, Inc., Chicago, IL, USA).

\section{Results}

\section{Baseline Study Characteristics}

A total of 432 patients were included in this study (mean age 57.2 years; $50.2 \%$ male), of whom $53(12.3 \%)$ had advanced DKD. The baseline characteristics of the advanced and nonadvanced DKD groups are shown in Table 1. The advanced DKD group was older, had a longer duration of diabetes, and higher percentages of HTN, PAOD, retinopathy, and neuropathy. There were no significant differences in gender, BMI, the prevalence of coronary artery disease, cerebral vascular accidents, smoking, and family history of diabetes between the 2 groups. The average UACR and triglyceride levels were significantly higher in the advanced DKD group, while the average levels of eGFR, low-density lipoprotein (LDL) cholesterol, and alanine aminotransferase were significantly higher in the nonadvanced DKD group. 
Table 2. Variables showing prognostic significance for advanced DKD

\begin{tabular}{|c|c|c|c|c|}
\hline Parameter & Beta coefficient & Standard error & Odds ratios $(95 \% \mathrm{CI})$ & $p$ value \\
\hline \multicolumn{5}{|l|}{ Univariate logistic regression } \\
\hline Age & 0.104 & 0.021 & $1.109(1.064-1.157)$ & $<0.001$ \\
\hline DM duration (months) & 0.007 & 0.002 & $1.007(1.004-1.010)$ & $<0.001$ \\
\hline Male gender & -0.226 & 0.294 & $0.798(0.448-1.420)$ & NS (0.442) \\
\hline BMI & 0.027 & 0.031 & $1.027(0.966-1.092)$ & NS (0.396) \\
\hline HTN & 2.912 & 0.728 & $18.397(4.413-76.689)$ & $<0.001$ \\
\hline PAOD & 2.427 & 0.925 & $11.327(1.847-69.476)$ & 0.009 \\
\hline Retinopathy & 0.645 & 0.282 & $1.905(1.096-3.312)$ & 0.022 \\
\hline Neuropathy & 1.025 & 0.314 & $2.786(1.507-5.154)$ & 0.001 \\
\hline Smoking & -0.321 & 0.364 & $0.725(0.356-1.479)$ & NS (0.377) \\
\hline Family history of DM & -0.524 & 0.374 & $0.592(0.285-1.233)$ & NS $(0.162)$ \\
\hline $\mathrm{eGFR}\left(\mathrm{mL} / \mathrm{min} / 1.73 \mathrm{~m}^{2}\right)$ & -21.407 & 149.437 & $<0.001(0.000-0.000)$ & NS $(0.086)$ \\
\hline UACR $(\mathrm{mg} / \mathrm{g})$ & 0.001 & $<0.001$ & $1.001(1.000-1.002)$ & $<0.001$ \\
\hline LDL cholesterol (mg/dL [median]) & -0.001 & 0.005 & $0.989(0.979-0.999)$ & 0.026 \\
\hline Triglycerides (mg/dL [median]) & 0.003 & 0.001 & $1.003(1.001-1.004)$ & 0.003 \\
\hline ALT & -0.036 & 0.014 & $0.965(0.939-0.991)$ & 0.010 \\
\hline $\mathrm{HbA} 1 \mathrm{C}$ & 0.144 & 0.076 & $1.155(0.995-1.340)$ & NS $(0.058)$ \\
\hline ULWPA & 1.012 & 0.478 & $2.751(1.078-7.022)$ & 0.034 \\
\hline ULCPA & 0.754 & 0.194 & $2.126(1.453-3.110)$ & $<0.001$ \\
\hline LLWPA & 0.427 & 0.207 & $1.532(1.021-2.301)$ & 0.040 \\
\hline LLCPA & 0.813 & 0.172 & $2.255(1.611-3.157)$ & $<0.001$ \\
\hline \multicolumn{5}{|c|}{ Multivariate logistic regression (including all variables except neuropathy) } \\
\hline Age & 0.095 & 0.030 & $1.100(1.037-1.167)$ & 0.002 \\
\hline DM duration & 0.006 & 0.002 & $1.006(1.002-1.011)$ & 0.008 \\
\hline HTN & 2.725 & 1.044 & $15.257(1.970-118.175)$ & 0.009 \\
\hline Triglycerides (mg/dL [median]) & 0.005 & 0.002 & $1.005(1.002-1.008)$ & 0.001 \\
\hline LLCPA & 0.732 & 0.237 & $2.079(1.306-3.309)$ & 0.002 \\
\hline Constant & -12.907 & 2.371 & $<0.001$ & $<0.001$ \\
\hline \multicolumn{5}{|c|}{ Multivariate logistic regression (including all variables) } \\
\hline Age & 0.096 & 0.031 & $1.101(1.037-1.169)$ & 0.002 \\
\hline DM duration & 0.006 & 0.002 & $1.006(1.001-1.011)$ & 0.009 \\
\hline HTN & 2.634 & 1.043 & $13.924(1.803-107.512)$ & 0.012 \\
\hline Triglycerides (mg/dL [median]) & 0.005 & 0.002 & $1.005(1.002-1.008)$ & 0.001 \\
\hline LLCPA & 0.757 & 0.240 & $2.133(1.332-3.414)$ & 0.002 \\
\hline Constant & -12.870 & 2.373 & $<0.001$ & $<0.001$ \\
\hline
\end{tabular}

Values in bold are statistically significant $(p<0.05)$. ALT, alanine transaminase; BMI, body mass index; DM, diabetes mellitus; DKD, diabetic kidney disease; HTN, hypertension; eGFR, estimated glomerular filtration rate; HbA1C, glycated hemoglobin; LDL, low-density lipoprotein; LLCPA, lower limb cold perception; LLWPA, lower limb warmth perception abnormality; PAOD, peripheral artery occlusive disease; UACR, urine albumin-to-creatinine ratio; ULCPA, upper limb cold perception abnormality; ULWPA, upper limb warmth perception abnormality.

There were no significant differences in the levels of total cholesterol, high-density lipoprotein cholesterol, $\mathrm{HbA1C}$, high-sensitivity C-reactive protein, and homocysteine between the 2 groups.

\section{Risk Factors for Advanced DKD}

Univariate analysis showed that age, diabetes duration, HTN, PAOD, retinopathy, neuropathy, UACR, LDL cholesterol, triglycerides, alanine aminotransferase, and upper and lower extremity warmth and cold perception abnormalities were good prognostic indicators for advanced DKD. To adjust for imbalances in baseline characteristics and multiple associa- 
Table 3. Correlation between the temperature perception abnormalities by QST and diabetes-associated adverse outcomes (Spearman rank correlation coefficients: $r$ )

\begin{tabular}{llllllllll}
\hline & ULWPA & ULCPA & LLWPA & LLCPA & PAOD & Retinopathy & Neuropathy & UACR & eGFR \\
\hline ULWPA & - & $0.299^{* *}$ & $0.281^{* *}$ & $0.271^{* *}$ & 0.049 & $0.132^{*}$ & $0.170^{* *}$ & 0.070 & $-0.193^{* *}$ \\
ULCPA & $0.299^{* *}$ & - & $0.315^{* *}$ & $0.407^{* *}$ & $0.132^{* *}$ & 0.039 & $0.172^{* *}$ & 0.107 & $-0.247^{* *}$ \\
LLWPA & $0.281^{* *}$ & $0.315^{* *}$ & - & $0.382^{* *}$ & 0.031 & $0.100^{* *}$ & $0.179^{* *}$ & 0.043 & $-0.211^{* *}$ \\
LLCPA & $0.271^{* *}$ & $0.407^{* *}$ & $0.382^{* *}$ & - & 0.054 & $0.158^{* *}$ & $0.189^{* *}$ & $0.152^{* *}$ & $-0.204^{* *}$ \\
PAOD & 0.049 & $0.132^{* *}$ & 0.031 & 0.054 & - & 0.047 & 0.057 & -0.024 & $-0.115^{* *}$ \\
Retinopathy & $0.132^{*}$ & 0.039 & $0.100^{*}$ & $0.158^{* *}$ & 0.047 & - & 0.089 & -0.005 & $-0.146^{* *}$ \\
Neuropathy & $0.170^{* *}$ & $0.172^{* *}$ & $0.179^{* *}$ & $0.189^{* *}$ & 0.057 & 0.089 & - & 0.076 & $-0.136^{* *}$ \\
UACR & 0.070 & 0.107 & 0.043 & $0.152^{* *}$ & -0.024 & -0.005 & 0.076 & - & $-0.282^{* *}$ \\
eGFR & $-0.193^{* *}$ & $-0.247^{* *}$ & $-0.211^{* *}$ & $-0.204^{* *}$ & $-0.115^{*}$ & $-0.146^{* *}$ & $-0.136^{* *}$ & $-0.282^{* *}$ & - \\
\hline
\end{tabular}

eGFR, estimated glomerular filtration rate; LLCPA, lower limb cold perception abnormality; LLWPA, lower limb warmth perception abnormality; PAOD, peripheral artery occlusive disease; QST, quantitative sensory testing; UACR, urine albumin-tocreatinine ratio; ULCPA, upper limb cold perception abnormality; ULWPA, upper limb warmth perception abnormality. ${ }^{*} p<$ $0.05 .{ }^{* *} p<0.01$.

tions between temperature perception abnormalities in the extremities and other variables, we used 2 multivariate models to evaluate the impact of temperature perception abnormalities in the extremities of patients with advanced DKD. The results showed that age, diabetes duration, HTN, triglycerides, and LLCPA had independent prognostic significance for advanced DKD (Table 2). Regression coefficients of these variables were used to calculate the odds of advanced DKD in each patient as follows:

Logarithm of the odds of advanced DKD $=$

$-12.870+0.757 \times$ LLCPA $+0.096 \times$ age $+0.006 \times$ diabetes duration $+2.634 \times \mathrm{HTN}+0.005 \times$ triglycerides.

\section{Correlations among Sensory Abnormalities in the Extremities and Diabetes-Associated} Complications

Spearman's rank correlation analysis revealed that all extremity thermal perception abnormalities were significantly negatively correlated with eGFR and that ULWPA, LLWPA, and LLCPA were significantly positively correlated with retinopathy. However, only LLCPA was significantly positively correlated with UACR (Table 3). The correlations between the severity of thermal perception abnormality and the features associated with advanced DKD, including eGFR, UACR, BMI, LDL, HbA1C, and HTN, are shown in Figure 2. It was observed that the degree of FLWCPA was positively correlated with that of UACR, HbA1C, and HTN, while negatively correlated with that of eGFR, BMI, and LDL.

\section{Discrimination of the Results of QST Tests}

Comparisons between the discriminatory abilities of the extremity sensory abnormalities determined by QST tests and other diabetes-associated complications are shown in Table 4. The discriminatory powers of FLCPA and FLWCPA were similar to that of UACR and significantly higher than those of PAOD and retinopathy. Among all the extremity abnormalities, AUROC analysis showed that FLCPA had the best discriminatory power.

The prevalence of temperature perception abnormalities among different CKD stages is shown in Figure 1. A progressive and significant increase in the prevalence of all extremity sensory abnormalities was correlated with increasing CKD stage of the patients. Among all extremity temperature perception abnormalities, ULWPA had the highest prevalence in our patients. In further comparisons, FLWPA had a higher prevalence than FLCPA, while ULWCPA had a higher prevalence than LLWCPA. 
Table 4. Pairwise comparison of AUROC for prediction of advanced DKD using the results of QST and diabetes-associated adverse outcomes

\begin{tabular}{|c|c|c|c|c|c|c|c|}
\hline Variable & AUROC & $95 \% \mathrm{CI}$ & $p$ value & $\begin{array}{l}\text { AUROC } \\
\text { difference }\end{array}$ & SE & $95 \% \mathrm{CI}$ & $p$ value \\
\hline ULWPA & 0.568 & $0.586-0.761$ & 0.032 & 0.262 & 0.054 & $0.068-0.301$ & $<0.001$ \\
\hline ULCPA & 0.672 & $0.806-0.913$ & $<0.001$ & 0.158 & 0.052 & $0.080-0.304$ & 0.003 \\
\hline LLWPA & 0.597 & $0.721-0.841$ & NS (0.137) & 0.233 & 0.055 & $0.168-0.381$ & $<0.001$ \\
\hline LLCPA & 0.718 & $0.811-0.911$ & $<0.001$ & 0.112 & 0.055 & $0.039-0.252$ & 0.043 \\
\hline FLWPA & 0.618 & $0.832-0.926$ & 0.009 & 0.212 & 0.054 & $0.157-0.368$ & $<0.001$ \\
\hline FLCPA & 0.741 & $0.861-0.939$ & $<0.001$ & 0.089 & 0.053 & $0.010-0.232$ & NS (0.094) \\
\hline FLWCPA & 0.729 & $0.648-0.810$ & $<0.001$ & 0.101 & 0.054 & $0.025-0.262$ & NS (0.061) \\
\hline PAOD & 0.510 & $0.414-0.606$ & NS (0.837) & 0.320 & 0.060 & $0.248-0.392$ & $<0.001$ \\
\hline Retinopathy & 0.597 & $0.498-0.695$ & 0.045 & 0.233 & 0.061 & $0.142-0.326$ & $<0.001$ \\
\hline Neuropathy & 0.589 & $0.493-0.686$ & NS (0.064) & 0.241 & 0.060 & $0.131-0.347$ & $<0.001$ \\
\hline UACR & 0.830 & $0.763-0.898$ & $<0.001$ & & & & \\
\hline
\end{tabular}

Values in bold are statistically significant $(p<0.05)$. AUROC, area under receiver operating characteristic; DKD, diabetic kidney disease; FLCPA, 4-limb cold perception abnormality; FLWCPA, combined 4-limb warmth and cold perception abnormality; FLWPA, 4-limb warmth perception abnormality; LLCPA, lower limb cold perception abnormality; LLWPA, lower limb warmth perception abnormality; PAOD, peripheral artery occlusive disease; QST, quantitative sensory testing; UACR, urine albumin-tocreatinine ratio; ULCPA, upper limb cold perception abnormality; ULWPA, upper limb warmth perception abnormality.

\section{Discussion}

In this study, the mean diabetes duration of our patients was 9.9 years, and the prevalence of neuropathy was $23.8 \%$, which is consistent with the findings of previous reports [28]. Among the 432 patients, $53(12.3 \%)$ had advanced DKD. Our analysis revealed that age, diabetes duration, HTN, triglycerides, and abnormal cold perception in the lower limbs were independent predictors of advanced DKD in the patients with T2DM (Table 2). Our results also showed that abnormal cold perception in the lower limbs was a good predictor of advanced DKD (AUROC 0.718). Moreover, abnormal cold perception in the 4 limbs had an even better discriminatory power, which was similar to that of UACR (AUROC 0.741 vs. 0.830 , $p=0.094$ ) and significantly better than that of PAOD, retinopathy, neuropathy, and other temperature perception abnormalities by QST (Table 4).

Previous investigations had reported that the duration of diabetes, age, triglycerides, and HTN were linked to the development and progression of diabetic small-fiber neuropathy [2931]. The correlations between these 4 factors and the occurrence of renal complications have also been well described in type 1 diabetes $[32,33]$. In the current study, we further demonstrated that these 4 variables were also independent predictors of advanced DKD in patients with T2DM (Table 2). Moreover, our findings confirmed the good discriminative power and independent predictive value of abnormal cold perception in the lower limbs for prediction of advanced DKD in these patients (Table 4). The correlations among all temperature perception abnormalities by QST were good, and the correlation between each temperature perception abnormality and eGFR was better than neuropathy (Table 3).

In this study, we also investigated the relationships between temperature perception abnormalities by QST and different stages of CKD in an attempt to elucidate whether temperature perception abnormalities by QST can be used as an early warning sign of DKD development. Our results showed that the prevalence of temperature perception abnormalities significantly increased with the progression of CKD (Fig. 1). DPN is characterized by progressive distal and symmetrical peripheral neuropathy of sensory nerve fibers, followed 


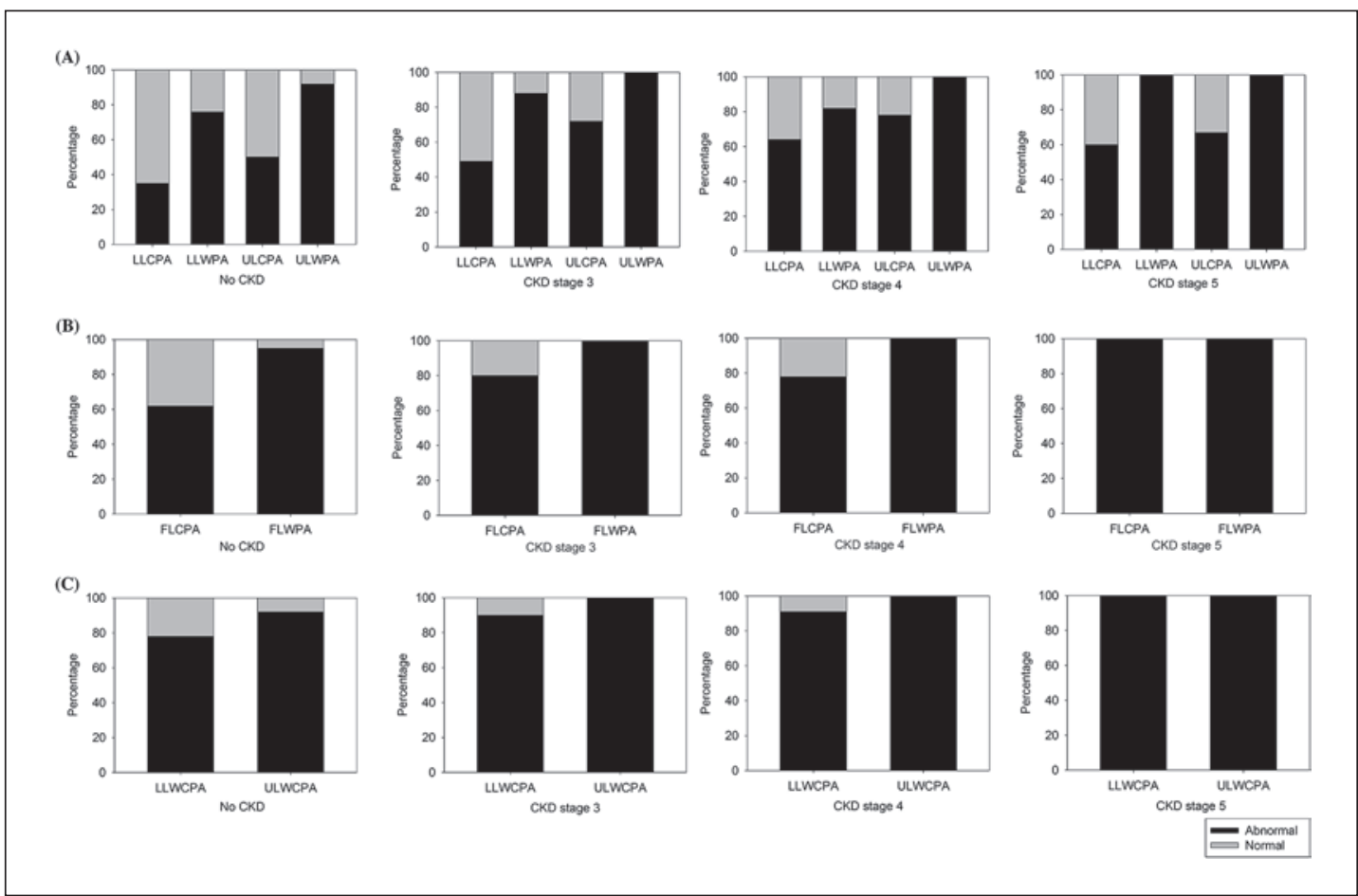

Fig. 1. The prevalence of temperature perception abnormalities by QST among different CKD stages. a Comparisons among LLCPA, LLWPA, ULCPA, and ULWPA. b Comparison between FLCPA and FLWPA. c Comparison between LLWCPA and ULWCPA. CKD, chronic kidney disease; FLCPA, 4-limb cold perception abnormality; FLWPA, 4-limb warmth perception abnormality; LLCPA, lower limb cold perception abnormality; LLWCPA, lower limb warmth and cold perception abnormality; LLWPA, lower limb warmth perception abnormality; QST, quantitative sensory testing; ULCPA, upper limb cold perception abnormality; ULWCPA, upper limb warmth and cold perception abnormality; ULWPA, upper limb warmth perception abnormality.

by autonomic and motor involvement [34]. Small-fiber neuropathy with abnormal thermal perception is the most frequent sensory impairment [35]. Abnormal cold perception can reflect dysfunction of myelinated Ad small fibers, and abnormal warmth perception can reflect dysfunction of unmyelinated C small fibers [12]. In this study, the prevalence of abnormal warmth perception was higher than that of abnormal cold perception in all 4 limbs. Our data are consistent with those of Weintrob et al. [36] who reported that unmyelinated C small fibers are more vulnerable than myelinated Ad small fibers in diabetes [12, 36]. Furthermore, previous studies have reported that diabetic small-fiber neuropathy is length dependent $[37,38]$. Longer nerves are more easily destroyed, so the distal nerves in the lower extremities are more vulnerable than those in the hands. Interestingly, our data showed that the prevalence of abnormal cold and warmth perception in the lower extremities was lower than that in the upper extremities by QST (Fig. 1). However, the difference in the prevalence of abnormal thermal perception between the upper and lower extremities was not significant in all different CKD stages. It is well known that multiple interrelationships exist among thermal perception abnormalities, and many potential confounders have been studied. Only abnormal cold perception in the lower extremities was found to be an independent predictor for advanced DKD after we performed multiple logistic regression analysis.

QST is a simple, reproducible, and noninvasive evaluation tool that has high diagnostic validity for sensory abnormality, especially diabetic small-fiber neuropathy $[13,14]$. To 


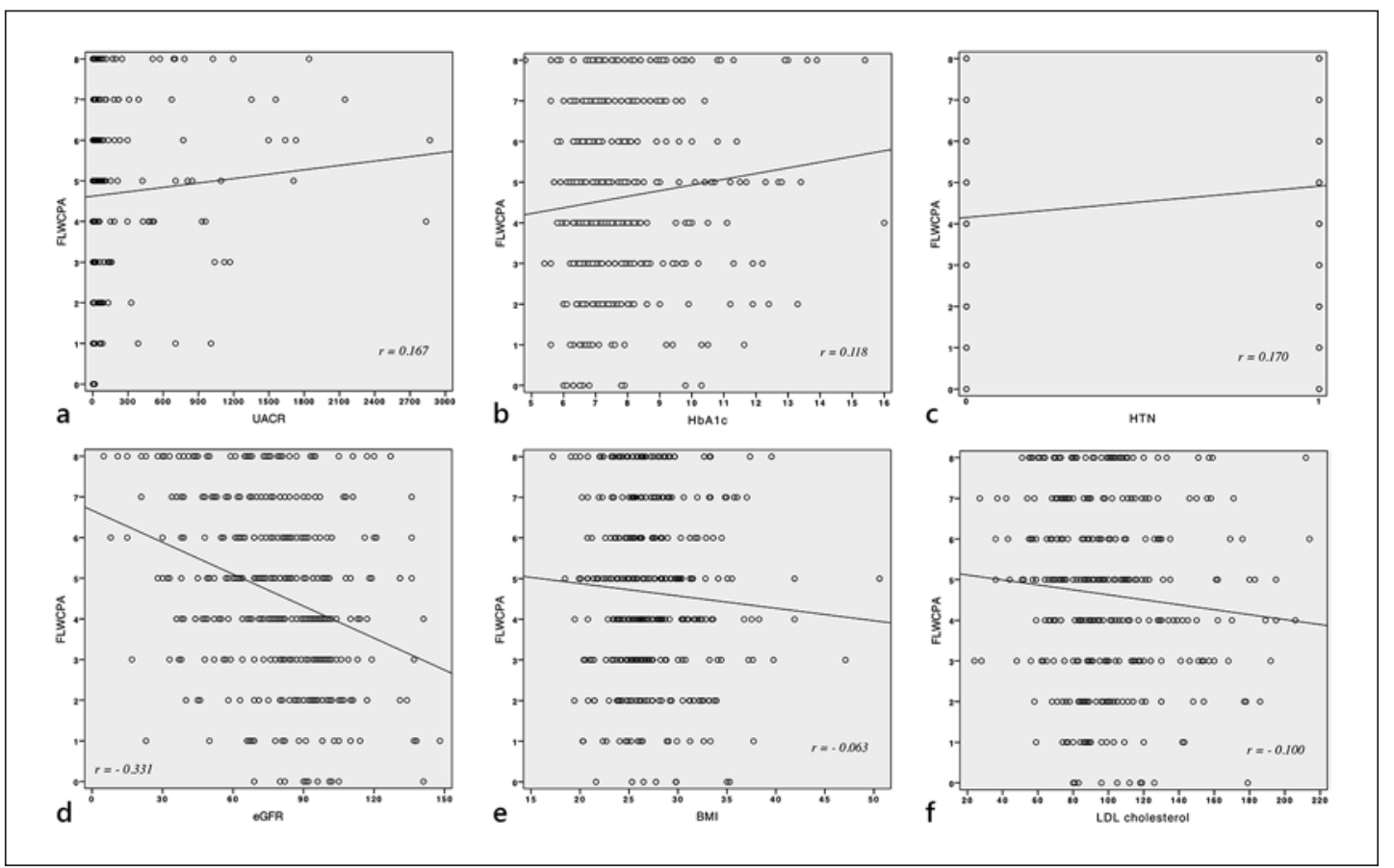

Fig. 2. The correlations between the severity of thermal perception abnormality and the features associated with advanced DKD. The overall trend of correlation between the degree of FLWCPA and features of advanced DKD, including (a) UACR ( $p=0.006)$, (b) HbA1C ( $p=0.054)$, (c) HTN $(p=0.001)$, (d) eGFR $(p<0.001)$, (e) BMI $(p=0.234)$, and (f) LDL ( $p=0.050)$. DKD, diabetic kidney disease; eGFR, estimated glomerular filtration rate; FLWCPA, 4-limb warmth and cold perception abnormality; HbA1C, glycated hemoglobin; HTN, hypertension; LDL, low-density lipoprotein; UACR, urine albumin/creatinine ratio.

the best of our knowledge, this is the first study to demonstrate that QST can help generate objective information for patients and physicians and aid in the early detection of advanced DKD. Patients with advanced DKD are known to have significant risks of various morbidities and mortality [39]. The early detection and timely referral to a nephrology specialist are critical and may aid in the prevention and/or management of these complications. These are important strategies to minimize suffering and the costs associated with this disease. Our study indicates that QST may be a valuable screening tool to identify individuals at risk of advanced DKD, especially in remote areas with a shortage of health resources.

In the past decades, emerging evidence has indicated central nervous system involvement in DPN, and the association between cognitive impairment and DPN in diabetes had been reported [40-42]. It is suggested that dysregulation of glycemic control may lead to microvascular dysfunction in the blood-brain barrier, autoimmune damage to neurologic diseases, and changes in the synthesis, availability, or reuptake of neurotransmitters. In the pathogenesis of DPN, there is an interaction between the peripheral pathway and the central pathway. A study investigating 36 type 2 diabetes patients revealed that the alteration of the central pathway of DPN documented by MRI may precede clinically evident cognitive impairment [40]. The presence and extent of albuminuria is associated with the cognitive changes in CKD [43]. In the current study, we confirmed that the prevalence of temperature perception abnormalities by QST increased with the progression of CKD, and the severity of temperature perception abnormality was also positively correlated to the degree of UACR 
and glycemic control, which might reflect endothelial damage (Fig. 2). Considering diabetes and CKD are correlated with increased risk of reversible cognitive dysfunction [41, 42], future personalized and specialized screening protocols for cognitive impairment in diabetes patients with thermal perception abnormality might help to allow timely interventions. Further well-powered research is needed to study this issue.

In spite of the encouraging results observed in this study, several potential limitations should be recognized. First, this study was conducted at a single medical center, and so the generalizability of our findings to other hospitals with different patient populations may be limited. Second, the results of the QST were based on subjective responses, so they were valid only when the patient was cooperative. Third, for lacking information about medication and diet control, we did not adjust these 2 important factors in the logistic regression models. Fourth, sequential measurements of thermal perception using QST (e.g., semiannually and annually) may reflect the dynamic aspects of clinical diseases and thus may have provided better information about the risk of advanced DKD risk in our patients. Finally, the predictive accuracy of logistic regression models has its own limitations.

In conclusion, the present study provides clinical evidence demonstrating an association between abnormal thermal perception by QST and an increased risk of advanced DKD in patients with type 2 diabetes. Our analysis also showed that abnormal cold perception in the lower limbs was an independent predictor of advanced DKD in these patients. Our results suggest that screening for DKD should start soon after the development of abnormal cold perception in the lower limbs by QST. Further prospective clinical studies with a larger sample size and long-term follow-up are warranted to validate the potential role of QST in reducing the burden on healthcare systems, especially in remote areas with a shortage of health resources.

\section{Acknowledgements}

The authors thank the staff of the Community Medicine Research Center of Keelung CGMH. They also express their sincere gratitude to all participants of the Taiwan Consortium for Acute Kidney Injury and Renal Diseases (CAKs).

\section{Statement of Ethics}

This study was conducted in full compliance with the ethical principles of the Declaration of Helsinki, the Good Clinical Practice guidelines, and the applicable local regulatory requirements. Subjects were invited to participate in this study when they visited our outpatient clinic. A trained endocrinologist examined all of the patients during screening and informed them about the consent procedure. Written informed consent was obtained from all subjects prior to their participation. This study was approved by the Institutional Review Board of Chang Gung Memorial Hospital (Approval No. 103-5291B).

\section{Conflict of Interest Statement}

The authors have no conflicts of interest to disclose. 


\section{Funding Sources}

This study was supported by the Chang Gung Memorial Hospital Research Program (CMRPG2F0171, CMRPG2F0172, CMRPG2F0173, CRRPG2H0161, CRRPG2H0162 and CMRPG2J0261) and the Ministry of Science and Technology of Taiwan (MOST 108-2321-B182-003 and MOST 109-2321-B-182-001).

\section{Author Contributions}

W.C.F., H.C.P., and K.M.C. contributed to the conception, design, and interpretation of data. W.C.F., K.M.C., and H.C.P. contributed to data collecting and manuscript drafting. C.Y.S., W.I.W., C.C.L., and Y.C.C. provided patient information, participated in the design and coordination, and helped draft the manuscript. C.C.L., C.Y.S., W.I.W., Y.C.C., and staff of the Community Medicine Research Center provided intellectual content for the work and were involved in editing and revising the manuscript. All authors discussed, contributed to, and approved the final manuscript version.

\section{References}

1 Guariguata L, Whiting DR, Hambleton I, Beagley J, Linnenkamp U, Shaw JE. Global estimates of diabetes prevalence for 2013 and projections for 2035. Diabetes Res Clin Pract. 2014;103(2):137-49.

2 Zimmet PZ, Magliano DJ, Herman WH, Shaw JE. Diabetes: a 21st century challenge. Lancet Diabetes Endocrinol. 2014;2(1):56-64.

3 Haw JS, Galaviz KI, Straus AN, Kowalski AJ, Magee MJ, Weber MB, et al. Long-term sustainability of diabetes prevention approaches: a systematic review and meta-analysis of randomized clinical trials. JAMA Intern Med. 2017;177(12):1808-17.

4 Zheng Y, Ley SH, Hu FB. Global aetiology and epidemiology of type 2 diabetes mellitus and its complications. Nat Rev Endocrinol. 2018;14(2):88.

5 Currie CJ, Poole CD, Woehl A, Morgan CL, Cawley S, Rousculp MD, et al. The financial costs of healthcare treatment for people with type 1 or type 2 diabetes in the UK with particular reference to differing severity of peripheral neuropathy. Diabet Med. 2007;24(2):187-94.

6 Brown JJ, Pribesh SL, Baskette KG, Vinik AI, Colberg SR. A comparison of screening tools for the early detection of peripheral neuropathy in adults with and without type 2 diabetes. J Diabetes Res. 2017;2017:1467213.

7 Callaghan BC, Cheng HT, Stables CL, Smith AL, Feldman EL. Diabetic neuropathy: clinical manifestations and current treatments. Lancet Neurol. 2012;11(6):521-34.

8 Papanas N, Ziegler D. Risk factors and comorbidities in diabetic neuropathy: an update 2015. The review of diabetic studies. Rev Diabet Stud. 2015 Spring-Summer;12(1-2):48-62.

9 Sheen YJ, Li TC, Lin JL, Tsai WC, Kao CD, Bau CT, et al. Association between thermal threshold abnormalities and peripheral artery disease in patients with type 2 diabetes. Medicine. 2018;97(51):e13803.

10 Shehab DK, Al-Jarallah KF, Abraham M, Mojiminiyi OA, Al-Mohamedy H, Abdella NA. Back to basics: ankle reflex in the evaluation of peripheral neuropathy in type 2 diabetes mellitus. QJM. 2011;105(4):315-20.

11 Juster-Switlyk K, Smith AG. Updates in diabetic peripheral neuropathy. F1000Res. 2016;5.

12 Jia ZR, Wang TT, Wang HX. Significance of quantitative sensory testing in the diagnosis of diabetic peripheral neuropathy. J Clin Neurophysiol. 2014;31(5):437-40.

13 Tavee J, Zhou L. Small fiber neuropathy: a burning problem. Cleve Clin J Med. 2009;76(5):297-305.

14 Ponirakis G, Odriozola MN, Odriozola S, Petropoulos IN, Azmi S, Fadavi H, et al. NerveCheck: an inexpensive quantitative sensory testing device for patients with diabetic neuropathy. Diabetes Res Clin Pract. 2016;113: $101-7$.

15 Lin CM, Yang MC, Hwang SJ, Sung JM. Progression of stages 3b-5 chronic kidney disease: preliminary results of Taiwan national pre-ESRD disease management program in Southern Taiwan. J Formos Med Assoc. 2013; 112(12):773-82.

16 Farrington K, Covic A, Nistor I, Aucella F, Clyne N, De Vos L, et al. Clinical practice guideline on management of older patients with chronic kidney disease stage $3 \mathrm{~b}$ or higher (eGFR $<45 \mathrm{~mL} / \mathrm{min} / 1.73 \mathrm{~m} 2$ ): a summary document from the European Renal Best Practice Group. Nephrol Dial Transplant. 2017;32(1):9-16.

17 Pinier C, Gatault P, François M, Barbet C, Longuet H, Rabot N, et al. Renal function at the time of nephrology referral but not dialysis initiation as a risk for death in patients with diabetes mellitus. Clin Kidney J. 2018; 11(6):762-8. 
18 Zhang Y, Jiang Y, Shen X, Yan S. Can both normal and mildly abnormal albuminuria and glomerular filtration rate be a danger signal for diabetic peripheral neuropathy in type 2 diabetes mellitus? Neurol Sci. 2017;38(8): 1381-90.

19 Xu L, Lin X, Guan M, Liu Y. Correlation between different stages of diabetic nephropathy and neuropathy in patients with T2DM: a cross-sectional controlled study. Diabetes Ther. 2018;9(6):2335-46.

20 Marathe PH, Gao HX, Close KL. American Diabetes Association Standards of Medical Care in Diabetes 2017. J Diabetes. 2017;9(4):320-4.

21 Levey AS, Coresh J, Greene T, Stevens LA, Zhang YL, Hendriksen S, et al. Using standardized serum creatinine values in the modification of diet in renal disease study equation for estimating glomerular filtration rate. Ann Intern Med. 2006;145(4):247-54.

22 Yun KJ, Kim HJ, Kim MK, Kwon HS, Baek KH, Roh YJ, et al. Risk factors for the development and progression of diabetic kidney disease in patients with type 2 diabetes mellitus and advanced diabetic retinopathy. Diabetes Metab J. 2016;40(6):473-81.

23 Paje D, Rogers MAM, Conlon A, Flanders SA, Bernstein SJ, Chopra V. Use of peripherally inserted central catheters in patients with advanced chronic kidney disease: a prospective cohort study. Ann Intern Med. 2019; 171(1):10-8.

24 Al-Qaisi M, Nott DM, King DH, Kaddoura S. Ankle brachial pressure index (ABPI): an update for practitioners. Vasc Health Risk Manag. 2009;5:833-41.

25 Fenner BJ, Wong RLM, Lam WC, Tan GSW, Cheung GCM. Advances in retinal imaging and applications in diabetic retinopathy screening: a review. Ophthalmol Ther. 2018;7(2):333-46.

26 Perkins BA, Orszag A, Ngo M, Ng E, New P, Bril V. Prediction of incident diabetic neuropathy using the monofilament examination: a 4-year prospective study. Diabetes care. 2010;33(7):1549-54.

27 Lysy Z, Lovblom LE, Halpern EM, Ngo M, Ng E, Orszag A, et al. Measurement of cooling detection thresholds for identification of diabetic sensorimotor polyneuropathy in type 1 diabetes. PLoS one. 2014;9(9):e106995.

28 Tsuji M, Yasuda T, Kaneto H, Matsuoka TA, Hirose T, Kawamori R, et al. Painful diabetic neuropathy in Japanese diabetic patients is common but underrecognized. Pain Res Treat. 2013;2013:318352.

29 Wiggin TD, Sullivan KA, Pop-Busui R, Amato A, Sima AA, Feldman EL. Elevated triglycerides correlate with progression of diabetic neuropathy. Diabetes. 2009;58(7):1634-40.

30 Nisar MU, Asad A, Waqas A, Ali N, Nisar A, Qayyum MA, et al. Association of diabetic neuropathy with duration of type 2 diabetes and glycemic control. Cureus. 2015;7(8):e302.

31 Ponirakis G, Petropoulos IN, Alam U, Ferdousi M, Asghar O, Marshall A, et al. Hypertension contributes to neuropathy in patients with type 1 diabetes. Am J Hypertens. 2019;32(8):796-803.

32 Perkins BA, Ficociello LH, Silva KH, Finkelstein DM, Warram JH, Krolewski AS. Regression of microalbuminuria in type 1 diabetes. N Engl J Med. 2003;348(23):2285-93.

33 Alleyn CR, Volkening LK, Wolfson J, Rodriguez-Ventura A, Wood JR, Laffel LM. Occurrence of microalbuminuria in young people with type 1 diabetes: importance of age and diabetes duration. Diabet Med. 2010;27(5): 532-7.

34 Perkins BA, Olaleye D, Zinman B, Bril V. Simple screening tests for peripheral neuropathy in the diabetes clinic. Diabetes care. 2001;24(2):250-6.

35 Chao CC, Hsieh SC, Yang WS, Lin YH, Lin WM, Tai TY, et al. Glycemic control is related to the severity of impaired thermal sensations in type 2 diabetes. Diabetes Metab Res Rev. 2007;23(8):612-20.

36 Weintrob N, Amitay I, Lilos P, Shalitin S, Lazar L, Josefsberg Z. Bedside neuropathy disability score compared to quantitative sensory testing for measurement of diabetic neuropathy in children, adolescents, and young adults with type 1 diabetes. J Diabetes Complicat. 2007;21(1):13-9.

37 Rader AJ, Barry TP. Symmetry of sensory loss in developing diabetic sensory polyneuropathy. Foot Ankle Spec. 2009;2(1):16-21.

38 Heldestad V, Linder J, Sellersjö L, Nordh E. Reproducibility and influence of test modality order on thermal perception and thermal pain thresholds in quantitative sensory testing. Clin Neurophysiol. 2010;121(11): 1878-85.

39 Brugnara L, Novials A, Ortega R, De Rivas B. Clinical characteristics, complications and management of patients with type 2 diabetes with and without diabetic kidney disease (DKD): a comparison of data from a clinical database. Endocrinol Diabetes Nutr. 2018 Jan;65(1):30-8.

40 Li J, Zhang W, Wang X, Yuan T, Liu P, Wang T, et al. Functional magnetic resonance imaging reveals differences in brain activation in response to thermal stimuli in diabetic patients with and without diabetic peripheral neuropathy. PLoS One. 2018;13(1):e0190699.

41 Ding X, Fang C, Li X, Cao YJ, Zhang QL, Huang Y, et al. Type 1 diabetes-associated cognitive impairment and diabetic peripheral neuropathy in Chinese adults: results from a prospective cross-sectional study. BMC Endocr Disord. 2019;19(1):34.

42 Viggiano D, Wagner CA, Blankestijn PJ, Bruchfeld A, Fliser D, Fouque D, et al. Mild cognitive impairment and kidney disease: clinical aspects. Oxford University Press; 2020.

43 Viggiano D, Wagner CA, Martino G, Nedergaard M, Zoccali C, Unwin R, etal. Mechanisms of cognitive dysfunction in CKD. Nat Rev Nephrol. 2020:16(8):452-69. 